\title{
Recurrent Sphenocavernous Meningioma
}

\author{
Mizuho Inoue ${ }^{1}$ Mohamed Labib ${ }^{2} \quad$ Alexander Yang ${ }^{1}$ \\ ${ }^{1}$ Department of Neurosurgery, University of Colorado, Aurora, \\ Colorado, United States \\ 2 Department of Neurosurgery, Barrow Neurological Institute, St. Joseph's \\ Hospital and Medical Center, Phoenix, Arizona, United States \\ ${ }^{3}$ Departments of Neurosurgery and Otolaryngology, University of \\ Colorado, Aurora, Colorado, United States
}

\author{
A. Samy Youssef ${ }^{3}$
}

J Neurol Surg B Skull Base 2022;83(suppl S3):e610.

\begin{abstract}
Address for correspondence A. Samy Youssef, MD, PhD, Departments of Neurosurgery and Otolaryngology, University of Colorado, Mail Stop C307, 12631 East 17th Avenue, Aurora, CO 80045, United States (e-mail: samy.youssef@cuanschutz.edu).
\end{abstract}

\begin{abstract}
A case of a recurrent sphenocavernous meningioma is presented. The patient is a 42-year-old male who presented with an episode of transient right-sided numbness. A magnetic resonance imaging (MRI) revealed a large left sphenocavernous meningioma. The patient underwent a frontotemporal craniotomy for tumor resection. Near total resection was achieved with minimal residual in the left cavernous sinus (CS) and orbital apex. The pathology was consistent with meningioma, World Health Organization (WHO) grade I. A follow-up MRI was done 9 months after surgery and showed a growth of the residual tumor, which was treated with intensity modulated radiotherapy. Tumor growth was detected on serial imaging over a 4-year period. Surgical resection was offered. A left frontotemporal craniotomy with pretemporal transcavernous approach was performed. The bone flap was reopened and the dura was opened in a Y-shaped fashion. The roof of the optic canal was drilled off, and the falciform ligament was opened to decompress the optic nerve. The tumor was disconnected from the anterior clinoid region (the anterior clinoid process was eroded by

Keywords

- recurrent

- sphenocavernous

- meningioma

- cavernous sinus the tumor) and reflected off the wall of the lateral CS. Tumor was adherent to the V2 fascicles (the lateral CS wall was resected in the first surgery) and was sharply dissected off. Gross total resection was achieved. The pathology was consistent with meningioma, WHO grade I. The patient had an unremarkable postoperative course without any new neurological deficits. The link to the video can be found at: https://youtu.be/KVBVw_86JqM.
\end{abstract}

Conflict of Interest

A.S.Y. is a consultant for Stryker Inc. and obtains royalties from Mizuho America Inc.

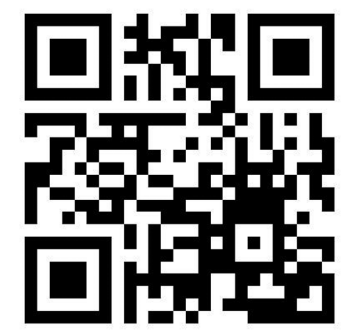

received

October 19, 2020

accepted

October 27, 2020

published online

June 8, 2021 www.thieme.com/skullbasevideos

www.thieme.com/jnlsbvideos
DOI https://doi.org/ 10.1055/s-0041-1725941. ISSN 2193-6331.

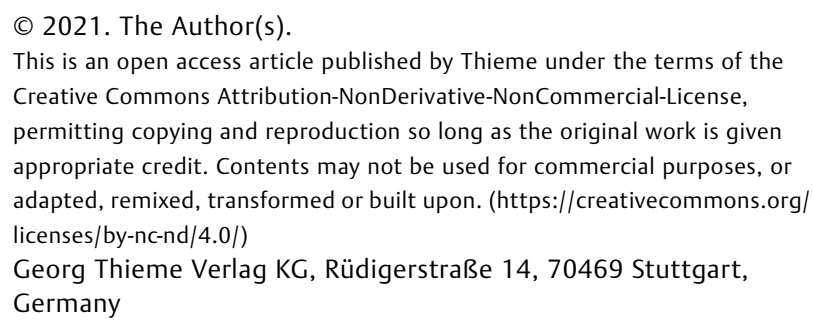

